\title{
Completion of Superconducting Magnet Production at BNL for the HERA Luminosity Upgrade
}

\author{
P. Wanderer, M. Anerella, J. Escallier, A. Ghosh, A. Jain, A. Marone, J. Muratore, B. Parker, A. \\ Prodell, P. Thompson, and K. C. Wu
}

\begin{abstract}
${ }^{\prime}$ Abstract-Brookhaven National Laboratory (BNL) has completed production of the superconducting multi-function magnets that are now installed as part of the HERA luminosity upgrade at DESY. The magnets, cryostats, and lead assemblies were designed and built at BNL. To fit inside the existing detectors, the coils plus cryostat structure had to meet a challenging radial budget (e.g., $39 \mathrm{~mm}$ horizontally). Two types of magnets were needed and three of each type were built. Each magnet contained normal and skew quadrupole, normal and skew dipole, and sextupole coils. The magnets operate in the $\sim 1.5 \mathrm{~T}$ solenoid field of a detector. The quadrupole coils produce gradients up to $13 \mathrm{~T} / \mathrm{m}$. The dipole coils generate fields up to 0.3 T. Coils were wound under computer control using either sevenstrand round cable or a single strand. To simultaneously avoid excessive synchrotron radiation background scattered from the beam pipe and yet have a small cryostat, one type of magnet used a tapered coil structure. The cryogenic system incorporates cooling with both $40 \mathrm{~K}$ helium and supercritical helium. All of the coils were tested in liquid helium in a vertical dewar. Quench test results have been excellent. The field quality of the magnets has met the stringent requirements imposed on interaction region magnets. One magnet of each type was tested at BNL as a completed assembly to verify the performance of the leads and cryostats. Two of each type were tested at DESY and then installed in the Zeus and $\mathrm{H1}$ experiments. The remaining magnets are spares. Final results of quench testing, field quality measurements and cryogenic performance are reported.
\end{abstract}

Index Terms-accelerator magnets, computer controlled winding, insertion region magnets, superconducting magnets

\section{INTRODUCTION}

$\mathrm{T}$ HE design of the recently completed HERA Luminosity Upgrade at DESY requires the use of superconducting magnets inside the Zeus and $\mathrm{H} 1$ detectors [1]. Two types of magnets, denoted GO and GG, were used (Fig. 1). Both types have dipole and quadrupole layers, to provide final focussing for electrons and for electron proton beam separation. Both

Manuscript received September 24, 2001. This work was supported in part by the U.S. Department of Energy under Contract No. DE-AC0298CH10886. The authors are with Brookhaven National Laboratory. (A. Prodell and P. Thompson are recently retired from BNL.) P. Wanderer is at BNL, Building 902, Upton NY 11973 USA (wanderer@bnl.gov, telephone 1-631-344-7687). types also have skew dipole, skew quadrupole, and sextupole windings. However, they have different lengths, field strengths, and internal configurations. To be compatible with operation inside the solenoid magnets of the two experiments with minimal shadowing of the detector by the magnets, the magnets have no magnetic flux return yokes and are supported inside thin-walled small-diameter cryostats. Space restrictions imposed significant constraints on the design of magnets, endcans, and lead towers.

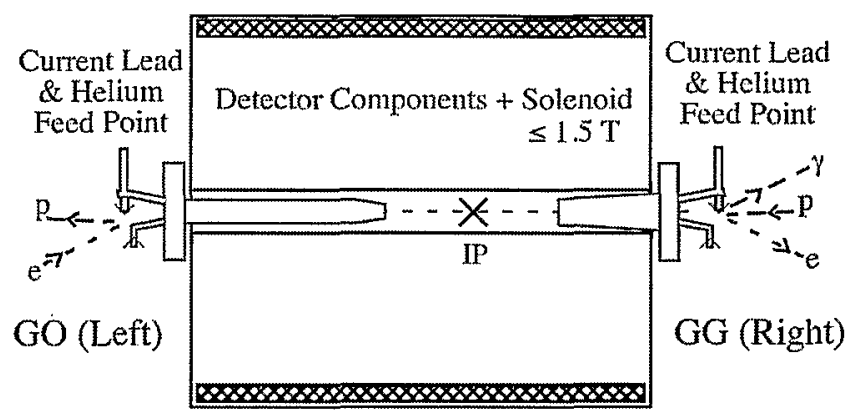

Fig. 1. Layout of superconducting magnets and detector solenoid.

A total of six magnets, including spares, were made. After a summary of the construction, this paper reports the test results for all the magnets. Detailed design and construction information can be found in earlier publications [2] -[4].

\section{II.MAGNET PRODUCTION}

\section{A: Magnet Design Parameters}

The magnets primarily affect the $27.5 \mathrm{GeV}$ electron or positron beam in HERA, since its momentum is much less than that of the $920 \mathrm{GeV}$ proton beam. Because of the synchrotron radiation, different magnets were needed for the incident and outgoing beams. The optics design of the IR called for five superconducting elements in both types of magnets, as indicated in Table I. The elements for each magnet were wound on a single support tube.

Some of the magnet requirements were typical for IR magnets: significant margin in operating current, operation in a solenoid field (1.5 T), higher order multipoles at most a few $\mathrm{x} 10^{-4}$ of the fundamental field, low heat leak, magnet centers located at the $50 \mu \mathrm{m}$ level. The limited radial space 
available for superinsulation and cryostat supports (Fig. 1) made it challenging to meet this goal.

TABLE I - MAGNET PARAMETERS

\begin{tabular}{rcccccc}
\hline \multirow{2}{*}{ Circuit } & \multicolumn{3}{c}{ GO Magnets } & \multicolumn{3}{c}{ GG Magnets } \\
\cline { 2 - 7 } & $I(\mathrm{~A})$ & $\begin{array}{c}\text { Field }(\mathrm{T}) \\
\text { at 31 mm }\end{array}$ & $\begin{array}{c}L_{c f f} \\
(\mathrm{~m})\end{array}$ & $I(\mathrm{~A})$ & $\begin{array}{c}\text { Field }(\mathrm{T}) \\
\text { at } 45 \mathrm{~mm}\end{array}$ & $\begin{array}{c}L_{c f f} \\
(\mathrm{~m})\end{array}$ \\
\hline Quad & 505 & 0.410 & 3.1 & 500 & 0.159 & 1.33 \\
\hline Dipole & 332 & 0.168 & 3.1 & 311 & 0.304 & 1.19 \\
\hline Skew Quad & 150 & 0.038 & 1.4 & 37 & 0.025 & 1.04 \\
\hline Skew Dipole & 150 & 0.076 & 1.4 & 37 & 0.041 & 0.94 \\
\hline Sextupole & 20 & 0.005 & 2.6 & 16 & 0.006 & 0.90 \\
\hline
\end{tabular}

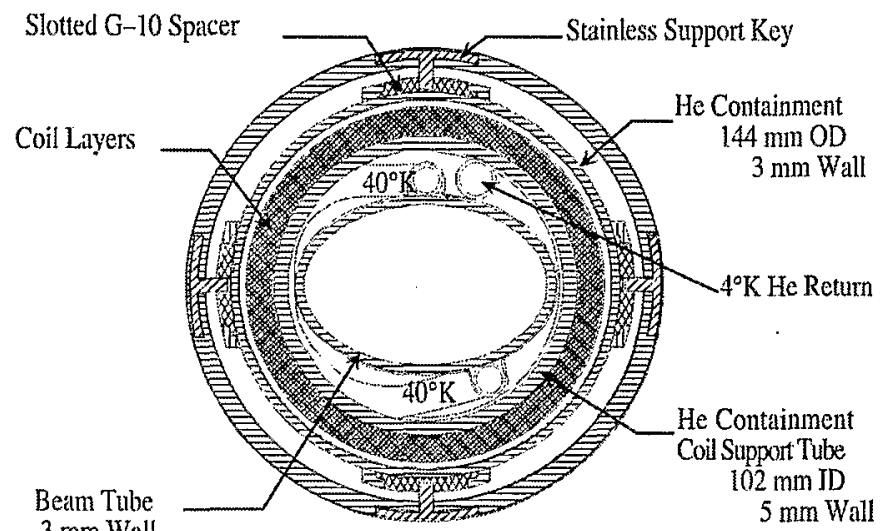

$3 \mathrm{~mm}$ Wall

Fig. 2. Cross section of GO magnet at internal support location.

Two novel requirements were placed on the magnets. The electron or positron beam is off center horizontally $(6 \mathrm{~mm}$ in $\mathrm{GO}, 20 \mathrm{~mm}$ in $\mathrm{GG}$ ), so the field quality was evaluated at the off-center positions, resulting in even tighter tolerances on conductor placement than usual. To avoid building new $\mathrm{H} 1$ detector components yet provide sufficient aperture for synchrotron radiation, the GG magnet was built with a taper $(10 \mathrm{~mm}$ in diameter over $1.4 \mathrm{~m})$.

\section{B. Superconductor}

Two configurations of superconductor were used. The superconductor used for the quadrupole and dipole windings was a round cable with six wires around a central wire. The wire diameter was $0.33 \mathrm{~mm}$ and the $\mathrm{Cu}: n o n-\mathrm{Cu}$ ratio was 1.8 . The short-sample limit of the cable was typically $615 \mathrm{~A} \mathrm{(5T}$, $4.5 \mathrm{~K})$. The diameter of the NbTi filaments was $8 \mu \mathrm{m}$.

The superconductor used for the skew quadrupole, skew dipole, and sextupole windings was a single wire $0.33 \mathrm{~mm}$ in diameter, with a Cu:non-Cu ratio of 2.5 , and a typical shortsample limit of $88 \mathrm{~A}(5 \mathrm{~T}, 4.5 \mathrm{~K})$.

Both conductors were insulated with overlapping layers of $25 \mu \mathrm{m} \mathrm{Kapton}{ }^{\mathrm{TM}}$ coated on the outside with B-stage epoxy. The conductors were similar to those used in RHIC magnets.

\section{Coil Design Software}

Three software packages developed at BNL were used to generate instructions for a sophisticated automatic winding machine. The first, COILGEN, optimized winding patterns for coils wound on a perfect cylinder (Fig. 2). Field uniformity at the $10^{-4}$ level was achieved in the design of the straight section and, separately, in the ends by varying the number of conductor blocks and the wire-to-wire spacing within each block. To reduce the number of splices in a layer, coil patterns could be generated starting at the inside turn or at the outermost turn. (Some coils have more than one layer. When layer " $n$ " was designed, the target harmonics were the negative of those measured on the previous layers, thus reducing the effect of construction tolerances.)

The second program transformed the COILGEN output to compensate for the measured offset of the center of the tube with respect to the axis of rotation. The largest offsets were in the range $0.5 \mathrm{~mm}-0.75 \mathrm{~mm}$. The resulting field quality was consistent with wire placement precision at the $50 \mu \mathrm{m}$ level.

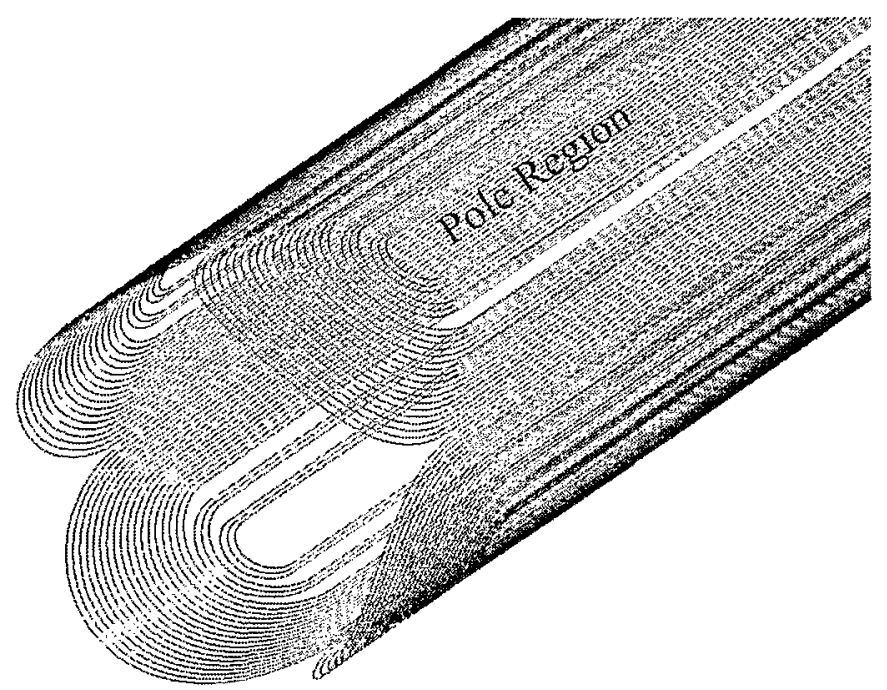

Fig. 3. End region of one GO quadrupole layer.

The third software package took the actual conductor positions and added process-dependent instructions, such as the power level of the ultrasound used to soften the epoxy prior to bonding. A total of 11 parameters (eight mechanical and three process variables) were used.

\section{Coil Manufacturing}

Coils were wound on a $316 \mathrm{~L}$ stainless steel support tube with a $5 \mathrm{~mm}$ wall. The particular tubes purchased were selected for low permeability in the allowed range. For the tapered GG support tube, significant effort was required to arrive at a schedule of machining and annealing that did not distort the tube excessively. The support tube was spiral wrapped with Kapton for ground plane insulation and then with an adhesive substrate. After a layer was wound, it was measured at room temperature ("warm") with a current of $2 \mathrm{~A}$ ( $0.2 \mathrm{~A}$ for the elements wound with a single strand). Layers with poor field quality could be easily removed and rewound at this time. This was done once in production.

When a satisfactory layer had been wound, the spaces between coil blocks in the straight section and end were filled with G10 spacers. Smaller spaces, such as those between the 
Geneva, Switzerland, September 24-28, 2001.

wires, were filled with an epoxy resin that was loaded with alumina.

After this epoxy had cured, the coil was wrapped $(0.7$ turns $/ \mathrm{mm}$ ) with epoxy-loaded S-2 glass roving under $134 \mathrm{~N}$ tension to provide a coil prestress of approximately $0.15 \mathrm{MPa}$ (220 psi) per layer. This provided a margin of $\sim 50 \%$ against the calculated Lorentz force load maximum of $0.11 \mathrm{MPa}(166$ psi). The compression wrap increased the transfer function slightly (by moving the wires to smaller radius) but did not change harmonics. The wrapped coil was then clamped with four thick-walled machined quarter cylinders of pipe and cured to provide a firm, cylindrical surface for winding the next layer. Typically, the resulting cured coil was round within $\pm 50 \mu \mathrm{m}$.

To achieve the needed field strength, three layers were used for the GO quadrupole coil and two layers were used for the GG dipole coil.

\section{E. Cryostat and End Volume}

The beam tube was elliptical (Fig. 2) to provide space for the $40 \mathrm{~K} \mathrm{He}$ circuit that removes heat from synchrotron radiation and scattered particles. Copper braid was welded to the beam pipe and the He pipe for good heat transfer. The beam pipe was made from $3 \mathrm{~mm}$-thick Nitronic 50 stainless steel. One of the most difficult tasks in making the cryostat was forming the GG beam pipe, which was both elliptical and tapered.

The coil support tube formed the inner wall of the $\mathrm{He}$ containment vessel. The end flanges were welded to it before the coils were wound. The radial distance available for the coil, compression wrap, and helium flow was $16 \mathrm{~mm}$.

The outer wall of the helium vessel was Nitronic 50 stainless steel with wall thicknesses $3 \mathrm{~mm}$. The vacuum can was also Nitronic 50. The wall thickness of the GG can was 3 $\mathrm{mm}$. For GO, the wall thickness was $7 \mathrm{~mm}$ for the $\sim 70 \mathrm{~cm}$ section nearest the end can, and $3 \mathrm{~mm}$ for the remainder.

Lorentz forces are produced by the $1.5 \mathrm{~T}$ field of the solenoid acting on the ends of the dipole coil. The net radial force pushes the end of one half coil in and the other half out, resulting in a net force on the magnet end. The compression wrap transmits this force to the helium containment vessel. To limit coil motion relative to the cryostat, special keys were installed between the helium vessel and the cryostat can, at both ends of the magnets. Keys were also installed at the end of the GO trim dipole, near the axial center of GO. Each key has two parts (Fig. 2). A stainless steel "T" welded to the vacuum can slides in a slotted G10 piece secured to the outer wall of the He vessel. The horizontal keys restrain the vertical motion. The slot in the G10 accommodates the thermal contraction of the cold mass. The radial thickness of the slotted G10 was important in restricting the local heat leak so that no condensation occurred. A difference of $0.5 \mathrm{~mm}$ changed the temperature at the surface of the cryostat from $-16 \mathrm{C}$ to $+16 \mathrm{C}$. Eighteen layers of superinsulation were placed in the $9 \mathrm{~mm}$ radial space available for the vacuum region. The cold masses were secured to the end cans (away from the IP). The slots in the two-part keys allow axial motion during thermal cycling.
The cryostatted magnet is supported at just one end by the endcan. The end can is separated from the cryostatted magnet during an intermediate $\mathrm{Hl}$ installation step, so the connection for this magnet is bolted. For GG, the connection is welded. The axial space available for the end can was quite limited ( $300 \mathrm{~mm}$ for $\mathrm{GO}, 210 \mathrm{~mm}$ for $\mathrm{GG}$ ), crowding the space needed for the vacuum, supercritical $\mathrm{He}, 40 \mathrm{~K} \mathrm{He}$, current leads, and instrumentation wiring connections. For GG, it was necessary to use a rectangular, instead of round, conflat ring to close the lead box, a further complication. A round bellows made the connection between the elliptical beam tube and the warm, round beam pipe in the endcan. The bellows is shielded from beam image currents by RF-fingers that are long and thin to minimize the heat leak to the $40 \mathrm{~K}$ beam pipe.

\section{F. Extension Arm and Lead Tower}

Due to space constraints in the endcan region, the stabilized leads and instrumentation wiring pass through "extension arms" $\sim 2 \mathrm{~m}$ long to reach the lead towers. The He return is also through the extension arm.

Each lead tower contains four $1000 \mathrm{~A}$ and four $500 \mathrm{~A}$ commercial leads (Cryomagnetics, Oak Ridge, TN U.S.A.). Sextupoles are powered using wires carried through the instrumentation feedthroughs.

\section{CRYOGENIC TEST RESULTS}

\section{A. Cold Mass Test in Liquid Helium (4.5K)}

Coils were powered separately and then in combination to at least $10 \%$ above their nominal operating currents [4]. Trim coils were powered with the dipole and quadrupole windings at operating current. There were no spontaneous quenches.

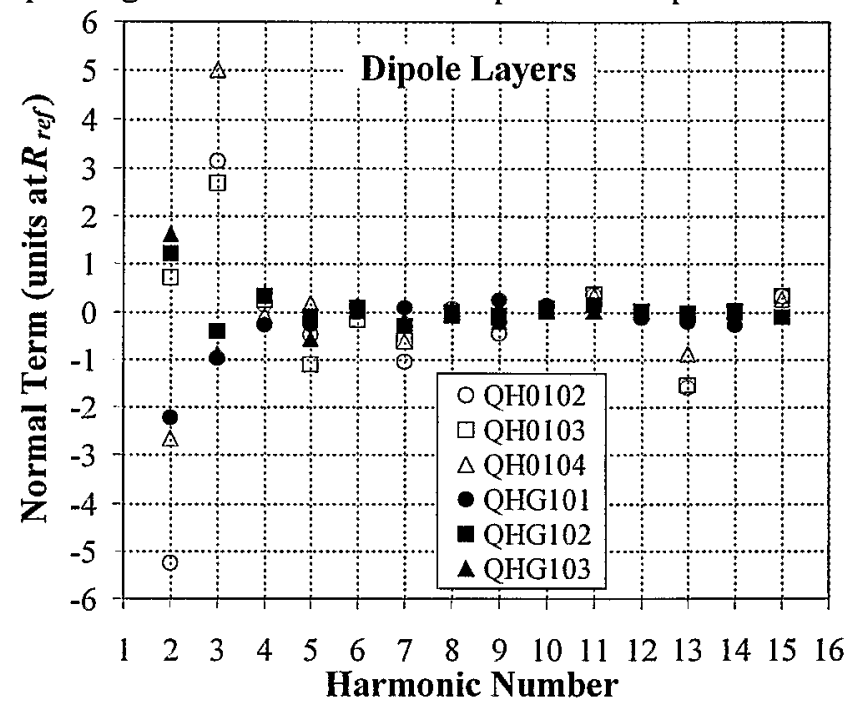

Fig. 4. Normal terms for the dipole layers of GO (QHO) and GG (QHG).

Before production started, a short test GO magnet with dipole and quadrupole layers was operated in a $1.5 \mathrm{~T}$ solenoid field to verify performance under solenoid-related loads. Quenches in each coil were artificially generated by a heater to check insulation and self-protection properties.

Harmonic measurements of the dipole and quadrupole coils in the GO magnets are shown in Fig. 4-7. A "unit" is $10^{-4}$ of 
Geneva, Switzerland, September 24-28, 2001.

the fundamental field, evaluated from the magnet center at the appropriate reference radius ( $31 \mathrm{~mm}$ for $\mathrm{GO}, 45 \mathrm{~mm}$ for $\mathrm{GG}$ ). Dipole terms correspond to $\mathrm{n}=1$. The harmonics were determined from the slope of the plots of harmonics against current, for increasing currents. (However, the quadrupole terms in the dipole layers are from warm measurements. Cold measurements of these terms could not be used due to the presence of unidentified magnetic materials near the dewar.) The normal and skew quadrupole terms and the normal sextupole can be compensated by other GO windings. The other terms are generally less than 1 unit. The reference radii given above include the horizontally-offset good field regions, so the field quality evaluated at the off-center regions was acceptable. The difference between the dipole and quadrupole field angles is less than $1 \mathrm{mrad}$. The centers of the dipole and quadrupole differ by less than $50 \mu \mathrm{m}$. Hysteresis measurements have been previously reported [4].

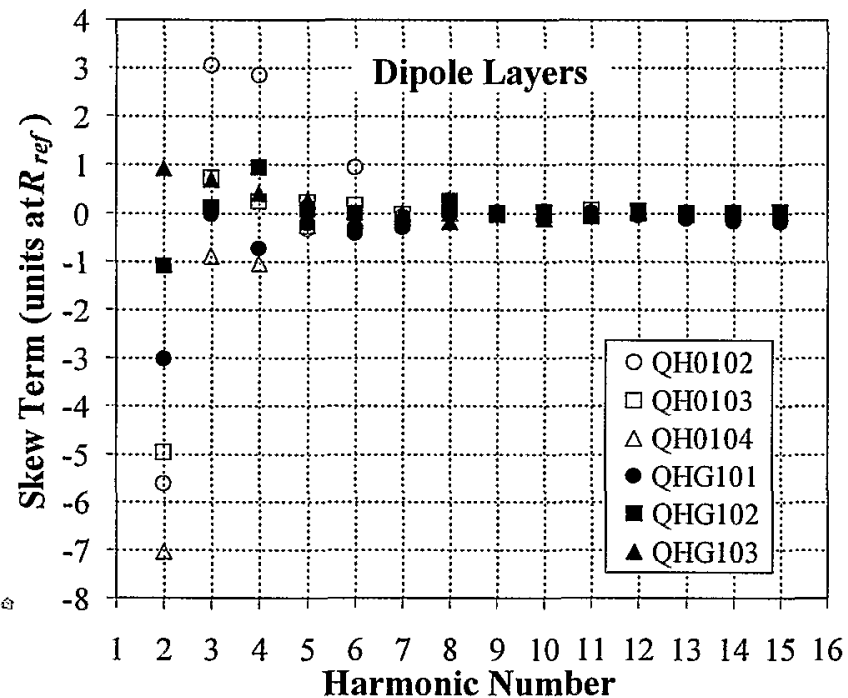

Fig. 5. Skew terms for the dipole layers of $\mathrm{GO}(\mathrm{QHO})$ and $\mathrm{GG}(\mathrm{QHG})$.

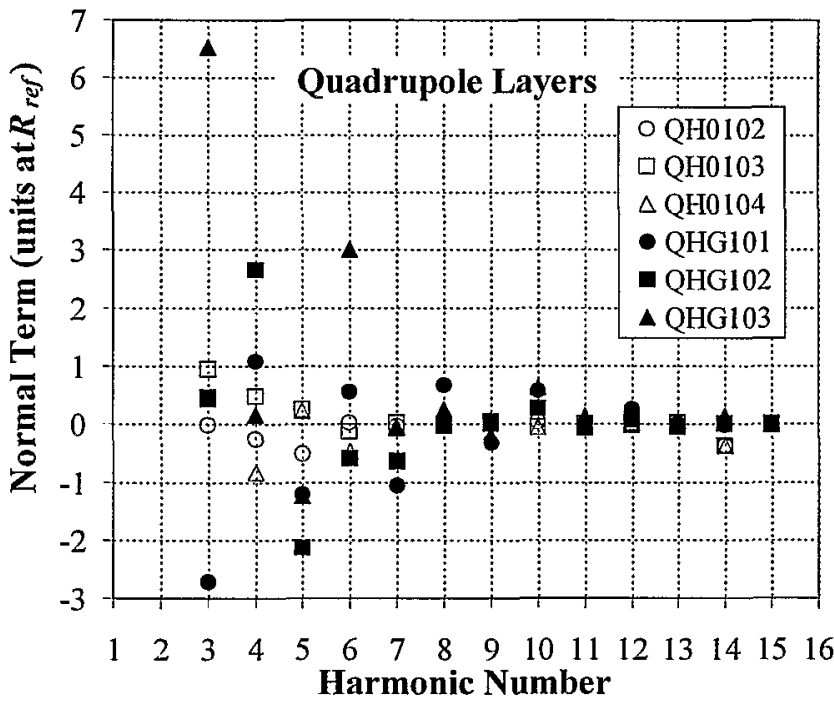

Fig. 6. Normal terms for the quad layers of $\mathrm{GO}(\mathrm{QH} 0)$ and $\mathrm{GG}(\mathrm{QHG})$.

\section{B. Tests of Completed Magnets}

The first completed GO and the first completed GG were tested in the Magcool test facility at BNL. The magnets were

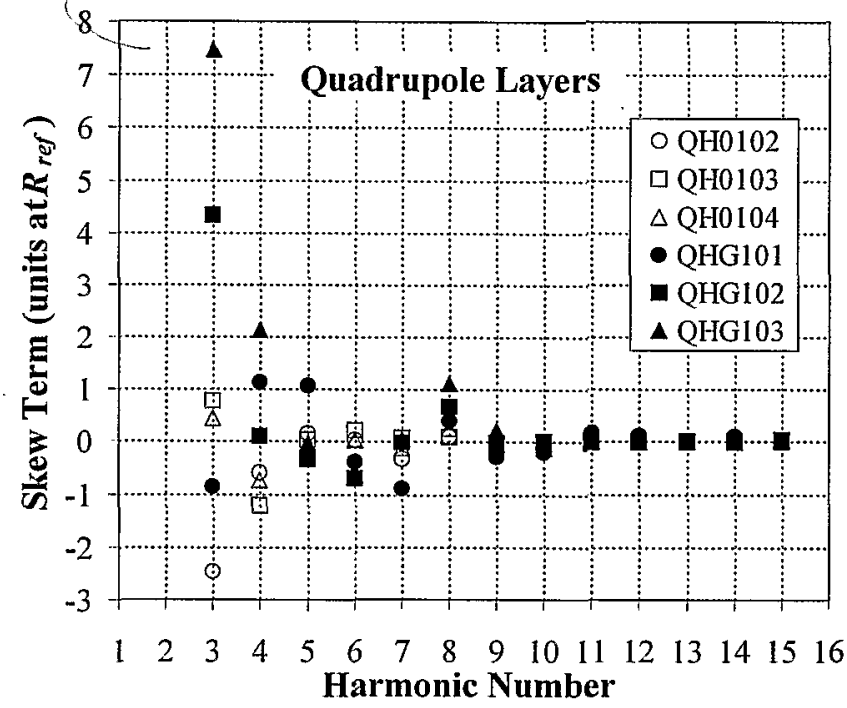

Fig. 7. Skew terms for the quad layers of $\mathrm{GO}(\mathrm{QHO})$ and $\mathrm{GG}(\mathrm{QHG})$.

powered in much the same way as during the tests in liquid He. No coil quenched. The heat load in the GG magnet was $30 \mathrm{~W}-40 \mathrm{~W}$. The heat load in the GO, less well determined, was less than $50 \mathrm{~W}$. ( $\left(\mathrm{LN}_{2}\right.$ was used instead of $40 \mathrm{~K}$ He in the beam tube cooling circuit, increasing the heat load.)

All completed magnets were measured using a "survey antenna" and surveyed at room temperature to determine the location of the quadrupole center with respect to fiducials on the cryostat. Measurements of the field center were made at seven positions along the magnets. The quadrupole axis in the first GO had a bow of $0.7 \mathrm{~mm}$. The bow was much less in the remaining magnets.

The first two GO and GG magnets were cold tested at DESY prior to installation. Their performance was satisfactory and the magnets were then installed in the experiments. The magnets have successfully operated in the $1.5 \mathrm{~T}$ solenoid fields of the experiments. The remaining two magnets are spares and lack extension arms and lead towers, precluding their test at DESY.

\section{CONCLUSIONS}

Special multilayer superconducting magnets have been successfully built for use in the HERA IR regions. Construction of these magnets required the development of new methods for designing and winding precision coils. Innovative engineering was required to prevent coil motion under Lorentz loading, while keeping tight budgets for heat leak and radial space.

\section{V.REFERENCES}

[1] M. Seidel, Luminosity upgrade of HERA, Proc. PAC99 .,pp. 34-36.

[2] B. Parker et al., Superconducting magnets for use inside the HERA interaction regions, Proc. EPAC98, pp 308-310.

[3] B. Parker et al., Test of a model superconducting magnet for the HERA ep interaction regions, Proc. MT16, in IEEE Trans. Applied Superconductivity vol. 10, no. 1, pp. 272-275, March 2000.

[4] B. Parker et al., HERA Luminosity upgrade superconducting magnet production at BNL, Proc. ASC2000 in IEEE Trans. Applied Superconductivity, vol. 11, no. 1, pp. 1518-1521, March 2001. 